\title{
New Method of Analyzing Adhesion/Friction in Resin-molded Structures and its Application to Insulated Rods*
}

\author{
Miki YAMAZAKI $^{* *}$, Satoshi IZUMI ${ }^{* * *}$ and Shinsuke SAKAI ${ }^{* * *}$ \\ ${ }^{* *}$ Mechanical Engineering Research Laboratory, Hitachi, Ltd. \\ 832-2 Horiguchi, Hitachinaka, Ibaraki 312-0034, Japan \\ E-mail: misuku.yamazaki.ra@hitachi.com \\ ${ }^{* * *}$ Department of Mechanical Engineering, School of Engineering, The University of Tokyo \\ 7-3-1 Hongo, Bunkyo-ku, Tokyo 113-8656, Japan
}

\begin{abstract}
The forces working at the contact interface between the metal and resin of a resin mold, which was made of metal cylinders covered with resin mold, were experimentally investigated. The forces discussed in this paper were modeled by friction force, adhesive force, and contact pressure based on residual stress. A new coefficient for adhesion was introduced by describing adhesion force. Moreover, FEM model of the forces acting on the contact interface was developed. The excellent agreement between the calculated and measured forces in applying the model to the design of an actual industrial product, i.e., an insulated rod, verified its accuracy.
\end{abstract}

Key words: Resin Molded Structure, Connected Interface, Friction, Adhesive Force, Residual Stress

\section{Introduction}

Resin materials are widely used for insulated rods and insulation vessels that are made of metal or ceramics cylinders covered with a resin mold because they have light-weight and high-intensity characteristics ${ }^{(1),(2)}$.

Frictional force, adhesive force, and face pressure act on the interface between metal or ceramic cylinders and resin in resin molds. These forces strongly influence the whole structure including that of the resin mold. A method of analysis that takes into consideration these forces in designing the interface configurations of resin-molded structures needs to be developed. However, the dynamic characteristics of the interface in the resin mold are still not completely understood. An adherence model that does not take the forces at the interface into consideration or only includes frictional force has usually been used.

The penalty method ${ }^{(3)}$ or the extended Lagrange method ${ }^{(4)}$ have often been used to analyze the contact problem, including frictional force at the interface. However, these techniques cannot be applied to a resin-molded structure to solve the contact problem while simultaneously taking into account the effect of frictional force, adhesive force, and surface pressure. Many researchers have aimed to solve such problems by studying cohesive-zone models ${ }^{(5)-(10)}$, which unify damage and exfoliation at the interface according to the principles of damage mechanics.

However, cohesive-zone models cannot serve as a standard model because of their complexity.

Given these issues, we devised a new model of forces acting on the interface between the metal and the resin. The model was derived by measuring the shear forces at the 
interface. In this study, we found that the shear force could be divided into three components, i.e., frictional force, adhesive force, and residual stress. Models based on a rigid-body spring and including the components of these three forces have been developed. These models were evaluated and verified by comparing simulated and measured fracture forces on the insulated rods of electric-power devices.

\section{Measurement of Shear Force at Interface}

The shear force at the interface between the metal and resin of a resin-molded structure in a metal cylinder and a resin mold was measured according to the procedure described below.

\subsection{Test pieces}

There is a photograph of the test piece, viz., a copper cylinder molded with resin, in Fig. 1. It was made by using a metallic mold into which a 10 -mm-thick resin was molded around a metal cylinder (37 $\mathrm{mm}$ in diameter and $27 \mathrm{~mm}$ in height). Three kinds of metal cylinders, i.e., oxygen-free high-conductivity copper (C1011), steel (SS400), or aluminum (A1050) were used.

To provide more detail, a liquid epoxy resin was poured into the metallic mold containing the metal cylinder, which then joined with the resin due to the temperature-hardening effect of the resin.

The temperature of the furnace during the molding process was fixed at a primary hardening temperature of $85^{\circ} \mathrm{C}$ for $7 \mathrm{~h}$ and a secondary hardening temperature of $130^{\circ} \mathrm{C}$ for $24 \mathrm{~h}$. After that, the test piece was cooled to room temperature $\left(20^{\circ} \mathrm{C}\right)$ at $7{ }^{\circ} \mathrm{C} / \mathrm{h}$.

Figure 2 plots the measured temperature in the resin during production of the test piece. It is clear that the maximum temperature difference $\left(110^{\circ} \mathrm{C}\right)$ and the highest temperature $\left(130^{\circ} \mathrm{C}\right)$ in Fig. 2 agree with those of the furnace settings. The glass-transition temperature of the epoxy resin we used was $140^{\circ} \mathrm{C}$. The viscous characteristics of the resin produced around the glass-transition temperature therefore had little influence.

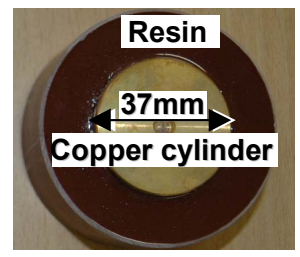

Fig. 1: Copper test piece

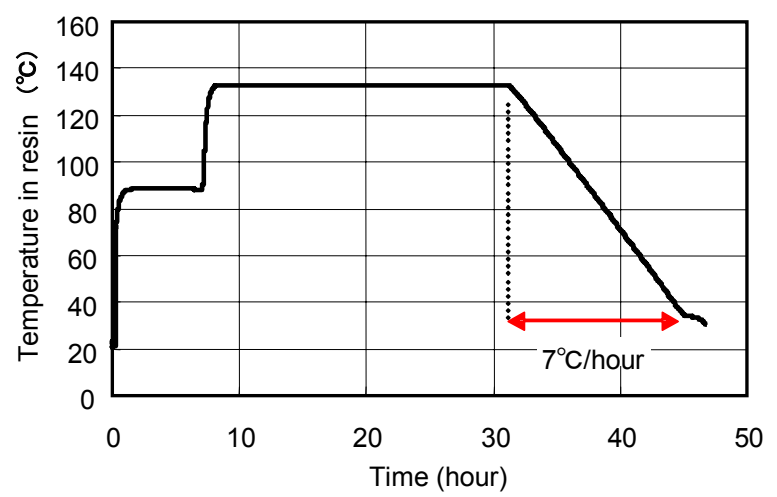

Fig. 2: Measured temperature in resin 


\subsection{Test method}

Figure 3 shows the experimental apparatus we used to measure the shear force at the metal-resin interface. A compression-testing machine (Shimadzu Autograph AG-100kNG) was used. The photograph in Fig. 4 shows the test piece installed on a jig. The jig had a hole so that the metal cylinder could be pushed out of the resin mold. The diameter of the hole was larger than the diameter of the metal cylinder. The ram of the testing machine pushed out the metal cylinder in the test of the resin mold at a rate of $0.01 \mathrm{~mm} / \mathrm{s}$.

Figure 5 has two photographs of the aluminum test piece. We measured the ram load i.e., shear force $f_{s}$, and displacement $x$ of the metal cylinder that had been pushed out. Two displacement sensors (A: CDP-25 and B: CDP-10, Tokyo Sokki Kenkyujo Co. Ltd.) were used. Sensor A measured the displacement at the tip of the ram. Sensor B measured the displacement in the jig. Displacement $x$ was a corrected value based on the difference between the outputs of Sensors A and B.

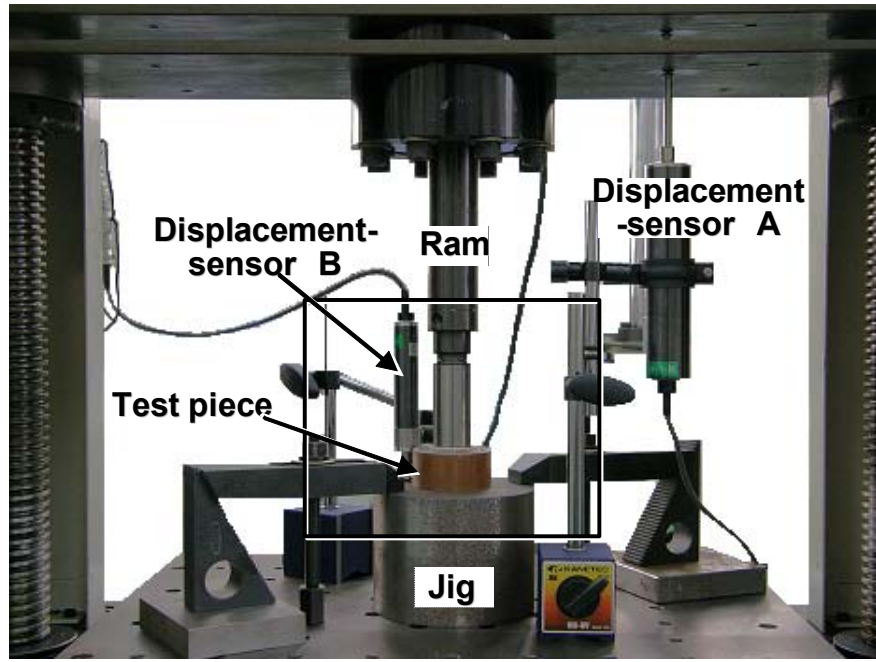

Fig. 3: Experimental system

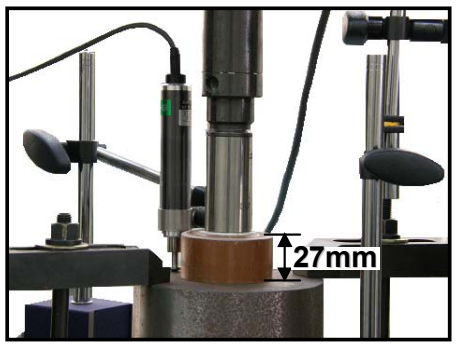

Fig. 4: Enlarged view around test piece

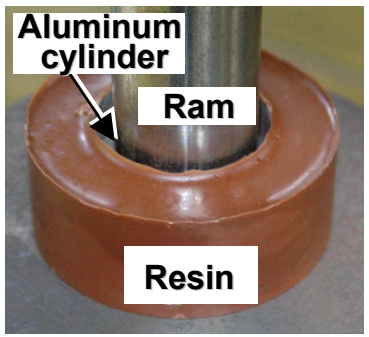

(a) Under test

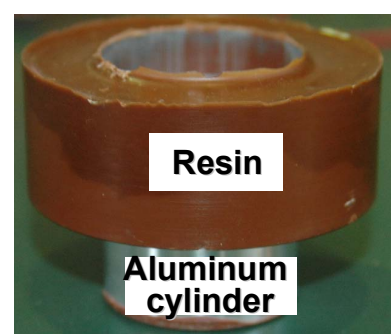

(b) Test piece for halfway test Fig. 5: Compression test 


\subsection{Test results on shear force}

Figure 6 shows the relation between measured shear force $f_{s}$ and displacement $x$ for the three test pieces made of copper, steel, and aluminum. Note that the displacement axis in Fig. 6 is on a logarithmic scale. For all three test pieces, the time histories of the shear force have two peaks in which the shear force quickly increases and immediately decreases.

This is considered because this structure is not a planar structure but an axially symmetrical structure. That is, if debonding occurs, adhesive force will be released, and the initial uniform pressure may redistribute. It is thought that a peak will appear while the adhesive force has concentrated locally and become stabilized. The first peak is considered to indicate the occurrence of debonding.

The highest peaks for shear force were $30 \mathrm{kN}$ for copper, $33 \mathrm{kN}$ for steel, and $8 \mathrm{kN}$ for aluminum.

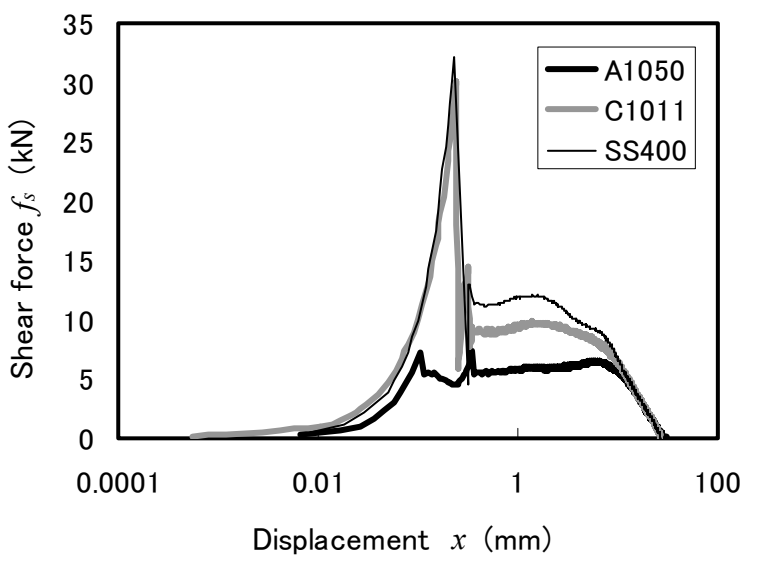

Fig. 6: Relationship between shear forces and displacement

\section{Modeling of Forces at Interface}

The forces acting on the interface had to be modeled as explained below to devise a suitable method of analyzing the experimental measurements for the resin mold presented in the preceding section.

\subsection{Forces acting on interface}

Figure 7 shows an axially symmetrical model of the copper test piece in Fig. 1. The initial condition, in which the metal cylinder and the resin were joined on the interface, is shown in Fig. 7(a) on the left. Figure 7(b) shows the deformed state in which axial displacement is applied to the top surface of the metal cylinder. The metal and the resin deforms as one body until the maximum shear force $f_{\text {SMAX. }}$. After that, the interface begins to crack.

Figure 8 plots the relation between displacement and shear strain at the interface, displacement and shear area, and displacement and shear force. As seen in Fig. 8(a), the metal and the resin deform together up to displacement $x_{e}$, at which the shear strain reaches $\gamma_{S}$ (i.e., $\left.x_{e} /\left(R_{2}-R_{I}\right)\right)$. For the shear area in Fig. $8(\mathrm{~b})$, the initial cross-sectional area, $A_{0}$, is constant up to $x_{e}$, at which the shear area decreases with further increase in $x$. Figure 8(c) plots the model of shear force $f_{s}$. Within the limits of shear force at the interface, $f_{s}$ can be determined by the product of shear stress $\tau_{s}$ and shear area $A_{0}$. Shear stress $\tau_{s}$ is obtained as the product of the shear modulus of elasticity $G$ and shear strain $\gamma_{s}(x)$. Shear force $f_{s}$ under elastic deformation $x_{e}$ therefore increases in proportion to $x$.

After shear force $f_{s}$ reaches maximum at $x=x_{e}$, further increments of $x$ reduce shear force $f_{s}$ within the shear area. The reason for this is that as shear force $f_{s}$ decreases rapidly, the metal and the resin are individually moved on the contact interface because the shear 
force exceeds the limit of shear strength as they detach.

It is assumed that the metal moves under linear uniform motion on the resin face when the metal and resin slip independently. Under this assumption, as the shear force equals the constant frictional force, the shear area should be decreased. The Coulomb-friction force can describe the shear force after the limit of shear strength is reached.

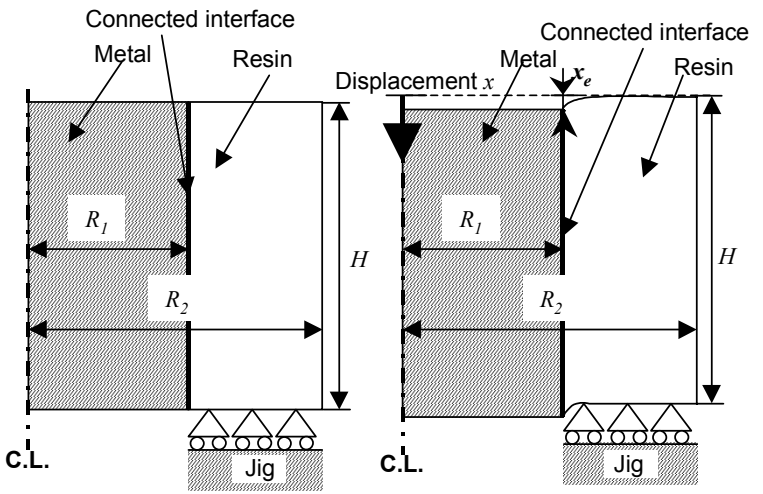

(a) Before test

(b) Test piece for halfway test

Fig. 7: Concept of test piece

(Axially symmetrical cross section)

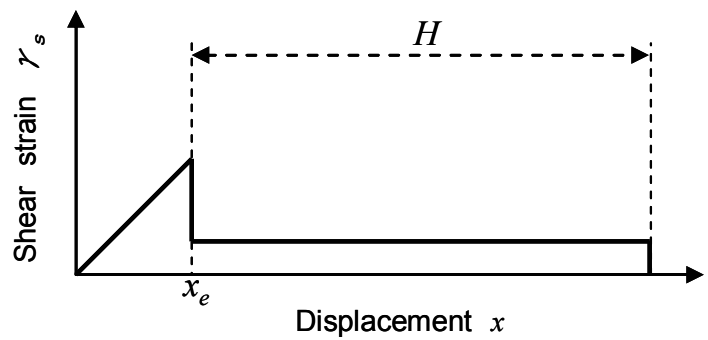

(a) Relationship between shear strain and displacement

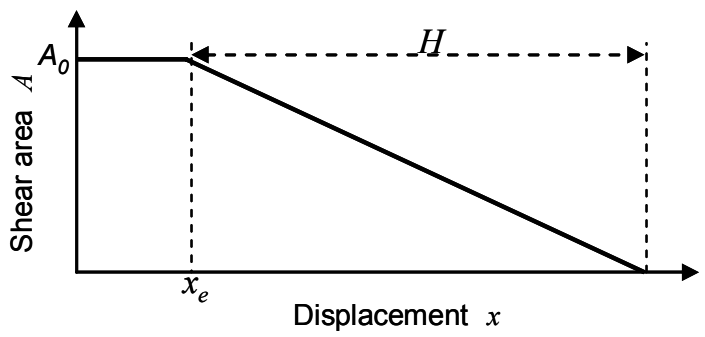

(b) Relationship between shear area and displacement

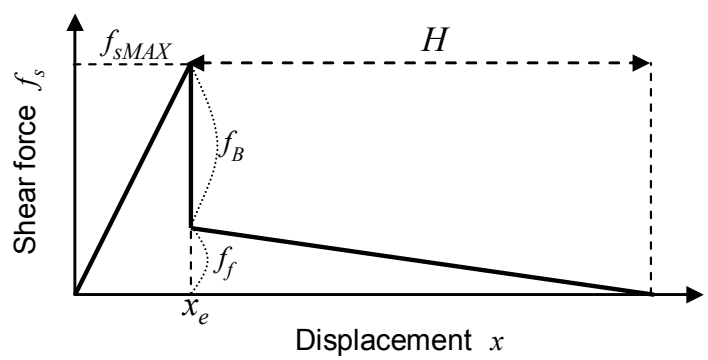

(c) Relationship between shear force and displacement Fig. 8: Modeling of contact interface 
This Coulomb-friction force is expressed in Fig. 8(c) where the shear stress at the limit of shear strength is expressed by $f_{S M A X}$; as a result, the total force (i.e., adhesive force $f_{B}$ added to friction force $f_{f}$ ) should agree with maximum shear force $f_{s M A X}$. Fictional force $f_{f}$ and adhesive force $f_{B}$ are considered to be dependent on pressure due to residual stress during molding.

The test results in Fig. 6 can be summarized as follows.

- Both frictional force $f_{f}$ and adhesive force $f_{B}$ act on the interface between the metal cylinder and the resin mold up to the limit of shear strength.

- When the metal cylinder begins to slide on the resin surface, adhesive force $f_{B}$ disappears, and only frictional force $f_{f}$ acts on the interface.

By comparing the materials in Fig. 6, the following conclusions could be drawn.

- The copper-resin mold had the largest adhesive force $\left(f_{B}\right)$ (i.e., $22 \mathrm{kN}$ ).

- The largest frictional force $\left(f_{f}\right)$ occurred for steel (i.e., $13 \mathrm{kN}$ ).

\section{Method of Analyzing Contact Based on FEM}

The forces acting on the interface between the metal and the resin described in the previous section were formulated as follows. First, shear-surface pressure was formalized according to shrink-fit theory. The friction and adhesive forces were formulated after that.

The formulated values were then applied to simulate the adhesion properties of the metal-resin interface. Finally, a new method of analyzing contact was developed.

\subsection{Surface pressure due to residual stress}

Molding resin onto a metal cylinder produces residual stress. A surface pressure of $\sigma_{p m}$ due to residual stress can be expressed by Eq. (1) according to shrink-fit theory for a combined cylinder,

$$
\sigma_{p m}=\frac{\delta}{2 R_{1}\left\{\frac{\left(1+v_{2}\right)\left(\frac{R_{2}}{R_{1}}\right)^{2}+\left(1-v_{2}\right)}{E_{2}\left(\frac{R_{2}}{R_{1}}\right)^{2}-E_{2}}+\frac{\left(1-v_{1}\right)}{E_{1}}\right\}}
$$

where $R_{1}$ and $R_{2}$ correspond to the outside diameters of the metal cylinder and resin, as shown in Fig. 7(a), $E_{1}$ and $E_{2}$ correspond to Young's modulus for the metal and resin and $v_{1}$ and $v_{2}$ correspond to their Poisson ratios, and $\delta$ is the shrink-fit clearance, which is determined by multiplying the difference in the expansions of the metal and the resin by temperature difference $\Delta T$.

\subsection{Frictional force}

Frictional force is expressed by the following formula based on a Coulomb friction model,

$$
f_{f}=\mu<-f_{n}>=\left\{\begin{array}{cc}
\mu f_{n} & \left(f_{n}<0\right) \\
0 & \left(f_{n}>0\right)
\end{array},\right.
$$

where $f_{f}$ is the tangential component of the friction force, and $f_{n}$ is the normal component of the surface pressure between the metal and the resin.

The degree of slide between the metal and the resin changes the cross-sectional area $A(x)$ of the interface between them and is expressed by

$$
A(x)=\left\{\begin{array}{cc}
2 \pi R_{2} H & \left(0 \leq x \leq x_{e}\right), \\
2 \pi R_{2}\left\{H-\left(x-x_{e}\right)\right\} & \left(x>x_{e}\right)
\end{array},\right.
$$

where $H$ is the height of the resin as shown in Fig. 4. The normal component of the surface 
pressure, $f_{n}$, and the friction force, $f_{f}$, are obtained from Eqs. (1) and (3) as

$$
f_{n}(x)=\sigma_{P m} A(x)
$$

and

$$
f_{f}(x)=\mu \sigma_{P m} A(x) .
$$

\subsection{Adhesive force}

Since the surface pressure due to residual stress acts normal to the interface, the normal reaction also works along the interface. The adhesive force produced on the interface is therefore proportional to the surface pressure and can be expressed as

$$
f_{B}=B<-f_{b}>=\left\{\begin{array}{cc}
B f_{b} & \left(f_{b}<0\right) \\
0 & \left(f_{b}>0\right)
\end{array},\right.
$$

where $f_{B}$ is the tangential component of adhesive force, and $f_{b}$ is the surface pressure (normal reaction force).

Note that $B$ is new in this paper and is referred to as the "adhesion coefficient." The adhesion coefficient does not depend on surface pressure or adhesion area; it describes the strength of adhesive bonding between the materials. As described in Section 3.1, the degree of slide between the metal and the resin decreases adhesive force $f_{B}$.

It is confirmed by the examination in which pressure was changed that the adhesive strength $f_{B}$ is proportional to pressure shown as Eq. (6). The following two influences are considered to cause this.

- Influence of the normal residual stress on the adhesion process.

- Improvement in the apparent shear strength of the interface by the effect of the mixed mode of the residual stress developed in the normal and shear directions.

Detailed observation of an interface and investigation into the mechanism are for future study.

Therefore, as seen in Fig. 8(c), when elastic deformation reaches $x_{e}$, adhesive force $f_{B}$ is zero. Adhesive force $f_{B}$ is then defined by using the step function as

$$
f_{B}(x)=\left\{\begin{array}{cc}
B \sigma_{P m} A(x) & \left(x \leq x_{e}\right) \\
0 & \left(x>x_{e}\right)
\end{array} .\right.
$$

\subsection{Method of analyzing contact based on FEM}

Based on the formulation derived in the previous section, a method of analyzing contact by simulating the adhesion characteristics at the interface with sufficient accuracy was devised as is explained below.

In the non-linear model of the finite element method, the two nodes of a contact pair are used to represent the nodes of the metal and the resin as shown in Fig. 9. The two nodes are then connected with a non-linear spring. It is assumed that the dynamics in the tangential and normal directions of the contact surface are independent. The shear spring is used for expressing deformation in the tangential direction, and the normal spring is used for that in the normal direction as shown in Fig. 9. In FEM, element division is performed so that contact interfaces have two nodes pair at equal intervals. Moreover, formula (8) holds for the whole system. (Spring constants are set so that the relation between total shear force and the elastic deformation follows eq. (8).) Furthermore, a normal spring uses the penalty rigidity which can be found from the Young's modulus of resin.

$$
f_{S}=\left\{\begin{array}{cc}
\left\{(\mu+B) \sigma_{p m} 2 \pi R_{1} H\right\} x & \left(x<x_{e}\right) \\
\mu \sigma_{p m} 2 \pi R_{1}\left\{H-\left(x-x_{e}\right)\right\} & \left(x>x_{e}\right)
\end{array} .\right.
$$




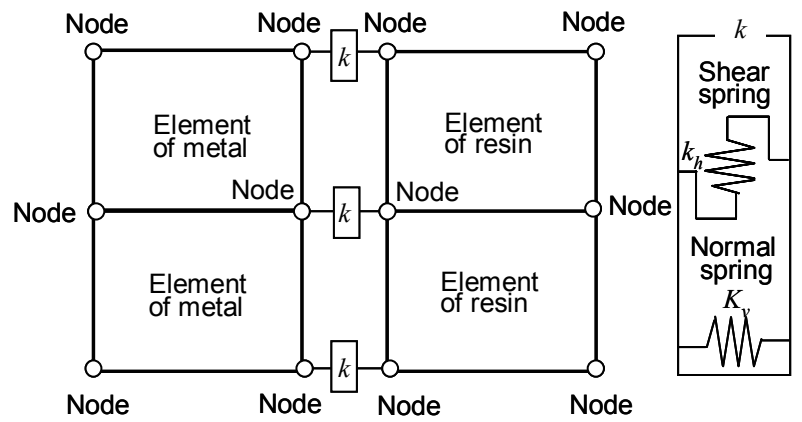

Fig. 9: Axially symmetrical model

\section{Verification of Accuracy of Adhesive Force and Adhesion Coefficient}

FEM, taking into account the adhesive force and adhesion coefficient described in the previous section, was used to analyze contact as is explained below. The accuracy of the method was then evaluated and verified by comparing the measured and simulation results.

\subsection{Definition of adhesion coefficient}

Maximum shear force at $x=x_{e}$ can be obtained from Eq. (8) as

$$
f_{\text {sMAX }}=(\mu+B) \sigma_{P m} A\left(x_{e}\right),
$$

where $\sigma_{P m}$ and $A(x)$ are calculated from Eqs. (1) and (3). The sum $(\mu+B)$ of friction coefficient $\mu$ and adhesion coefficient $B$ is obtained by substituting the shear force, $f_{S M A X}$, given by the measured results in Fig. 6 into Eq. (9). Friction coefficient $\mu$ is measured and adhesion coefficient $B$ can then be estimated.

Table 1 lists both the adhesion and friction coefficients. The adhesion coefficient is large, viz., in the order of that of copper, steel, and aluminum.

\subsection{Comparison of measured and simulation results}

Shear force $f_{s}$ was calculated by analyzing contact based on FEM described in Section 4 and was compared with the measured results in Fig. 6.

Table 1: Coefficients of friction and adhesion

\begin{tabular}{|c|c|c|c|}
\hline & $\mathrm{A} 1050$ & $\mathrm{C} 1011$ & $\mathrm{SS} 400$ \\
\hline \hline $\begin{array}{c}\text { Friction } \\
\text { coefficient } \mu\end{array}$ & 0.3 & 0.17 & 0.16 \\
\hline $\begin{array}{c}\text { Adhesion } \\
\text { coefficient } B\end{array}$ & 0.05 & 0.34 & 0.27 \\
\hline
\end{tabular}

Table 2: Material properties

\begin{tabular}{|c|c|c|c|c|c|}
\hline & Units & $\begin{array}{c}\text { Epoxy } \\
\text { resin }\end{array}$ & A1050 & C1011 & SS400 \\
\hline \hline Young's modulus & $\mathrm{GPa}$ & 15 & 70 & 117 & 206 \\
\hline Poisson's ratio & - & 0.29 & 0.35 & 0.34 & 0.3 \\
\hline $\begin{array}{c}\text { Thermal expansion } \\
\text { coefficient }\end{array}$ & $\mathrm{ppm} / \mathrm{K}$ & 52 & 23 & 17 & 14 \\
\hline
\end{tabular}




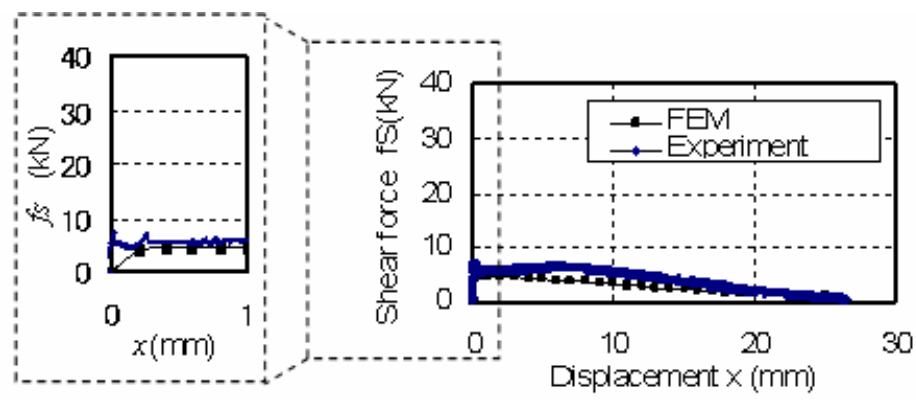

Fig. 10: Comparison of measured and numerical-analysis results (A1050)

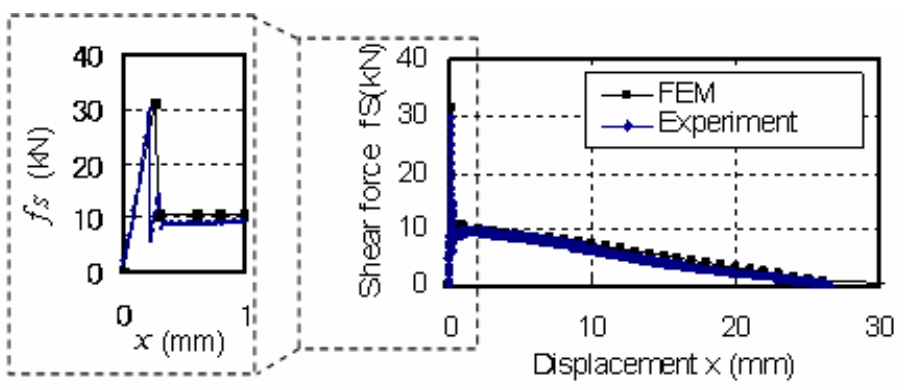

Fig. 11: Comparison of measured and numerical-analysis results (C1011)

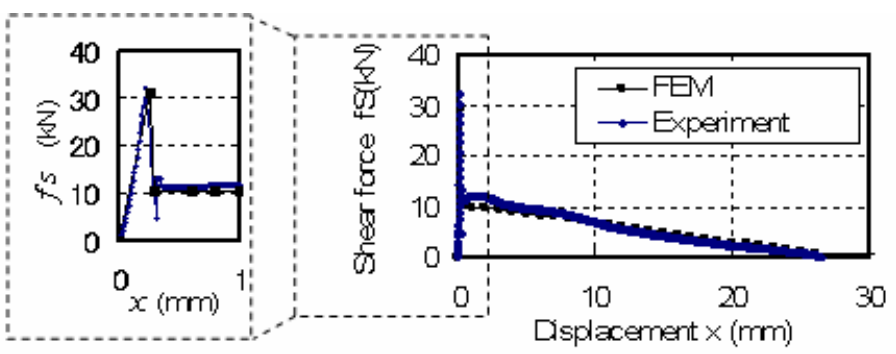

Fig. 12: Comparison of measured and numerical-analysis results (SS400)

The FEM model is an axially symmetrical model with an $R_{I}$ of $27 \mathrm{~mm}$, an $R_{2}$ of $37 \mathrm{~mm}$, and an $H$ of $27 \mathrm{~mm}$ as shown in Fig. 7. To simulate the test environment in Fig. 3, calculated residual stress was used to evaluate the surface pressure, and forced displacement was applied to the nodes on the top of the model shown in Fig. 7. The material properties are listed in Table 2. Frictional and adhesive forces were calculated from the surface pressure given by Eq. (1) by using the friction and adhesion coefficients listed in Table 1. After that, FE models were placed between the metal and resin of the non-linear spring models.

Both sets of results from Fig. 10 to Fig. 12 (i.e., measured and simulated) for the three metals were similar because the coefficients evaluated from the measured results were used.

\section{Application to Insulated Rods}

The stress on the insulated rods used in the switchgear of electrical-power devices was analyzed by using the technique we have proposed in this paper. The insulated rods, which are composed of two steel cylinders molded in resin, function to ground electrical-power devices .

\subsection{Finite element model}

Figure 13 shows an axially symmetric FEM of an insulated steel rod. The number of finite elements at the interface of the steel rod and the resin is the same as the number of set 
pairs of contact nodes. The adhesion coefficient $(0.27)$ and the coefficient of friction $(0.16)$ of SS400 steel listed in Table 1 were used. The material properties of the steel are listed in Table 2.

The non-linear spring is used for expressing deformation in the tangential direction, and the non-linear spring is used for that in the normal direction as shown in Fig. 9.

\subsection{Stress analysis}

The proposed method was verified by comparing the results of the stress analysis with or without considering the adhesive force at the interface. This model has the cross-sectional area of the normal direction of a resin interface smaller than the cross-sectional area of tangential direction. That is, it is a case where the tensile strength of an interface is lower than the shearing strength of the interface.

Figure 14 shows the relation between extensional force and maximum tensile stress. As for the result in the case that adhesive force was not considered (which is often the case), tensile stress reached the tensile strength $(43 \mathrm{MPa})$ of the resin at an extensional force of 15 $\mathrm{kN}$. On the other hand, in the case that the adhesive force was considered, the maximum tensile stress of the resin is reached at an extensional force of $63 \mathrm{kN}$. Therefore, in the result of this technique taking adhesive strength into consideration, when the maximum tensile stress of resin reaches the tensile strength $(43 \mathrm{MPa})$ of resin in the interface, the interface debonding and resin destruction occurs. Therefore, $63 \mathrm{kN}$ becomes a critical tensile stress of the interface strength.

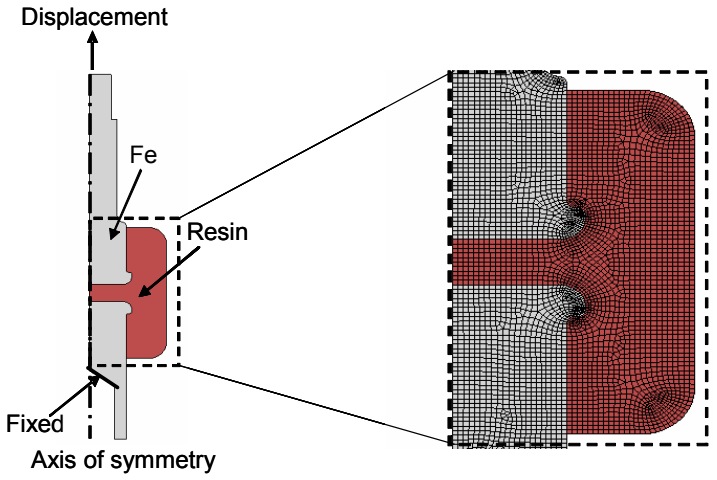

Fig. 13: Axially symmetrical model

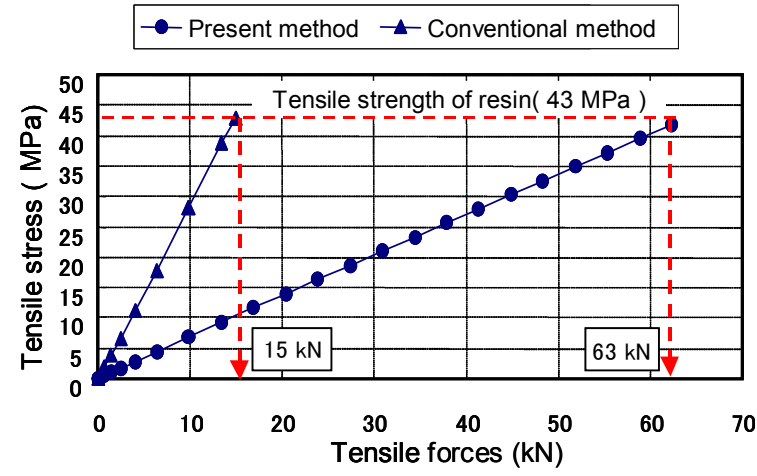

Fig. 14: Relationship between stress and forces 
The operating load of the insulated rod used is $16 \mathrm{kN}$. Therefore, the result means that the proposed method can reduce the size and cost of the insulating rod. On the other hand, according to this result obtained without considering adhesive force, the rod would have to be strengthened, thereby increasing its manufacturing cost. As a result, the enlargement and the high cost of the product is required by being based on the result without considering the adhesive force.

Moreover, it has been confirmed that the adhesion characteristic of the shear direction and normal direction could be expressed with this technique.

\subsection{Extensional Test}

We compared the extensional test results on the developed insulated rods and the results calculated with the method explained in Section 6.2. Three insulated rods were prepared. Displacement control at a speed of $0.01 \mathrm{~mm} / \mathrm{sec}$ was used to pull the steel cylinder, and it also measured the breaking forces. As a result, the three measured breaking forces were 64,68 , and $70 \mathrm{kN}$. These results were in excellent agreement with the breaking force of $63 \mathrm{kN}$ plotted in Fig. 13.

\section{Summary}

The forces working on the contact interface of a resin mold were modeled and formulated by using measured results. Simulation based on a formulization, viz., intensity analysis of insulated rods, was also carried out as follows.

First, deboning tests on the resin mold were carried out. Second, a model of the forces at the interface including the "adhesive force" was devised from the measured results. The adhesive force was formulized in terms of the "adhesion coefficient," which corresponded to the strength of adhesive bonding between the materials. Third, by comparing the simulation results with those we measured, we could evaluate and verify that the method was useful for estimating the crash stress in insulting rods.

\section{References}

(1) Okamoto, T., Shibuya, M., Takahashi, T., Mizutani,Y. and T., Takahashi, Proposal of All Solid Insulated Substation for Future Substation, IEEE 7th Intern. Conf. Properties and Application of Dielectric Materials (ICPADM), S22-4, (2003), pp. 1190-1193.

(2) Sato, J., Sakaguchi, O., Kubota, N., Makishima, S., Kinoshita, S., Shioiri, T., Yoshida, Miyagawa, M., Homma, M., and Kaneko, E., New Technology for Medium Voltage Solid Insulated Switchgear, IEEE/PES Transmission and Distribution Conference and Exhibition: Asia Pacific, Vol. 3, (2002), pp. 17911796.

(3) Yagawa, G., Hirayama, H., Miyoshi, A., and Ando, Y., Analysis of Contact Problem of Shell Structures Using Finite Element Method with Penalty Function, Transcations of the Japan Society of Mechanical Engineers. A, Vol. 48, No. 428, (1998), pp. 454-466.

(4) ABAQUS/Standard Ver. 6.4 User's Documentation.

(5) Shet, C., and Chandra, N., Analysis of Energy Balance When Using Cohesive Zone Models to Simulate Fracture Processes, Journal of Engineering Materials and Technology, Vol. 124, (2002), pp. 440-450.

(6) Alfano, G., and Sacco, E., Combining interface damage and friction in $\mathrm{q}$ cohesive-zone model, Int. J. Numer. Meth. Engng, No.68, (2006), pp. 542-582.

(7) Xu, X.P., and Needleman, A., Numerical simulations of dynamic crack growth along an interface, International Journal of Fracture, No. 74, (1996), pp. 289-324. 
(8) Dobert, C., Mahnken, R., and Stein, E., Numerical simulation of interface debonding with a combined damage/friction constitutive model, Computational Mechanics, No. 25, (2000), pp. 456-467.

(9) Needlman, A., A continuum model for void nucleation by inclusion debonding. J.App. Mech. 30, pp.259-276.

(10)Marotzke, C., and Qiao, L., Interfacial crack propagation arising in single-fiber pull-out tests, Comp. Sci. Tech. 57, (1997), pp. 887-897. 